\title{
NUMERICAL MODELLING OF HEAT TRANSFER DURING IMPACT OF A MOLTEN DROPLET ON A SURFACE
}

\author{
Roman O. Cherepanov ${ }^{1, *}$, and Alexander V. Gerasimov ${ }^{1}$ \\ ${ }^{1}$ EDP National Research Tomsk State University, 634050 Tomsk, Russia
}

\begin{abstract}
SPH-based numerical technique for modelling of impact of molten drops on a surface with heat transfer and phase transitions effects is proposed. Computational algorithm uses SPH with procedure of restoring of particle consistence and variational approach to calculation of acceleration field. Also, boundary algorithm for free and contact surfaces in $3 \mathrm{D}$ setting are developed.
\end{abstract}

\section{Introduction}

In plasma coating techniques metal powder is heated to a molten state and the flow of drops is directed onto the surface of tool. Molten droplets spread out over the surface, cool and solidify during interaction. The particles solidify to form a coating deposit on the substrate's surface. It is important to have a clear understanding of the physics of process and to have a models of droplet impact on the surface of substrates for better control of the generation of splats and the structure of coating. Experiments are used to get a link between factors related to droplet impact and final morphology and quality of coating, but this is an expensive way. Numerical models are more preferable for prediction of coating quality and properties, but it need a good computational model, able to treat high deformation, liquid flow and solidification, heat transfer and contact interaction of droplet and surface. Important side is ability to make modeling in $3 \mathrm{D}$, because of significant loose of information in simplified 2D-setting.

\section{Computational Method}

To calculate the parameters of splats, various approximation methods are used with different degrees of simplification. So, the representation of drops in the form of the equivalent cylinders provides a way to semi-analytical solution of the problem. Computational fluid dynamics or numerical method of solid mechanics lead to computer aided physical modeling in $3 \mathrm{D}$ setting.

\footnotetext{
${ }^{*}$ Corresponding author: rcherepanov82@gmail.com
} 
In this paper we propose the use of smooth particle method (SPH) [1], adapted to the modeling of the deformation process of the liquid droplet with a phase transition at crystallization in a large range of speeds.

Basis of SPH approximation is equation

$$
f^{i} \cong \int f(\vec{x}) W\left(\vec{x}-\vec{x}^{i}, h\right) d \vec{x}
$$

where $h$ is smoothing parameter, which define a radius of fluency for points, $\vec{x}-$ is a space coordinate, $W$ - smoothing function.

Spatial derivatives are defined via:

$$
f_{, \alpha}^{i}=\frac{\partial f^{i}}{\partial x_{\alpha}} \cong \int f(\vec{x}) W_{, \alpha}\left(\vec{x}-\vec{x}^{i}, h\right) d \vec{x}
$$

Corresponding to (2) particle approximation is written as:

$$
f_{, \alpha}^{i}=\frac{\partial f^{i}}{\partial x_{\alpha}} \cong \sum_{k} f^{k} W_{, \alpha}\left(\vec{x}^{k}-\vec{x}^{i}, h\right) \frac{m^{k}}{\rho^{k}}
$$

where $\vec{x}^{k}, f^{k}, m^{k}, \rho^{k}$ - radius-vector, approximated function value, mass and density at $k$-th point.

Equation (3) have $\mathrm{C}^{0}$-consistency [2] near surfaces and boundaries and at non-uniform particle distribution, and for restoring particle consistency special approaches needed. According [2], a test vector-function is introduced in form:

$$
\Delta(\vec{x})=\left(1, x_{0}, x_{1}, x_{2}\right)
$$

Approximation of test function (1) and its derivatives (2) can be found with $\mathrm{C}^{0}$ consistency, but due to the fact that this values are known, this approximation can be used to construct correction procedure and restore particle consistency. Let's define :

$$
\Delta(\vec{x})_{\alpha}=\left\{\begin{array}{l}
x_{\alpha} ; \alpha>-1 ; \\
1 ; \alpha=-1 ;
\end{array}\right.
$$

Matrix of approximations for this function at $n$-th point, found via uncorrected SPH approximation (3):

$$
T_{\beta \alpha}\left(\vec{x}^{n}\right)=\sum_{m} \Delta_{\alpha}\left(\vec{x}^{m}-\vec{x}^{n}\right) W_{, \beta}\left(\vec{x}^{m}-\vec{x}^{n}, h\right) \frac{m^{k}}{\rho^{k}} ;
$$

Lets define correction matrix as

$$
B_{\alpha \beta}^{n}=B_{\alpha \beta}\left(\vec{x}^{n}\right)=\left[T_{\alpha \beta}\left(\vec{x}^{n}\right)\right]^{-1} ; \alpha, \beta=-1,0,1,2 ;
$$

Now, corrected approximation of function $f(x)$ value or its derivatives can be found, if we build full matrix of uncorrected approximations (3) and apply correction matrix (7):

$$
f_{, \alpha}^{i}=\frac{\partial f^{i}}{\partial x_{\alpha}} \cong T_{\alpha \beta}^{i} \cdot\left\{\sum_{k} \frac{m^{k}}{\rho^{k}} f^{k} W_{, \beta}\left(\vec{x}^{k}-\vec{x}^{i}, h\right)\right\}
$$

For calculation of flow of liquid and solid phases, approximation (8) applied to lagrangian of system:

$$
L=\sum_{k} m^{k}\left(\frac{\vec{v}^{k} \vec{v}^{k}}{2}-u^{k}\right)
$$

where $\vec{v}^{k}$ - is particle speed, $u^{k}$ - is internal energy (per mass). In both phases (liquid and solid) internal energy change can be written in form:

$$
\frac{d u^{k}}{d t}=\sigma^{k}: \dot{\varepsilon}^{k}
$$


This implies:

$$
\frac{d L}{d t}=\sum_{k} m^{k}\left(v^{k} a^{k}-\sigma^{k}: \dot{\varepsilon}^{k}\right)
$$

Since the relation between a tensor of rate of deformation and velocity of nodes is linear:

$$
\dot{\varepsilon}^{k}=\frac{1}{2}\left(\left(\frac{\partial v_{i}^{k}}{\partial x_{j}}\right)+\left(\frac{\partial v_{i}^{k}}{\partial x_{j}}\right)^{T}\right)=S y m m_{-} \operatorname{part}\left(T_{j \beta}^{k} \sum_{n} v_{i}^{n} \frac{m^{n}}{\rho^{n}} W\left(\vec{x}^{n}-\vec{x}^{k}, h\right)_{, \beta}\right)
$$

(11) leads to a relation between the accelerations of nodes and stress field:

$$
a_{\gamma}^{n}=-\left(\sum_{k} \frac{m^{k}}{\rho^{k}} \sigma_{\gamma j}^{k} T_{j \beta}^{k} W_{, \beta}^{k n}\right) / \rho^{n}
$$

For calculus of heat transfer (8) is applied to heat transfer equation [3]

$$
\frac{d T}{d t}=\frac{-\left(\lambda T_{, j}\right)_{, j}+Q}{C_{P} \rho}
$$

Heat production of liquid is caused by viscous stress and defined as:

$$
Q_{\text {liquid }}=\sigma: \dot{\varepsilon}
$$

Heat production of solid is caused by plastic strain:

$$
Q_{\text {solid }}=\sigma: \dot{\varepsilon}^{p l}
$$

where $\dot{\varepsilon}^{p l}$ - rate of plastic strain, found via Mizes yield condition. For calculation of phase transition during solidification, latent heat is introduced to equation (14) via additional heat source, which operates when material cooled to temperature of melting.

Boundary condition for heat transfer is heat insulation on free surfaces and ideal heat contact at droplet-surface interface. For impact problem we use a condition of zero normal stress at free surface and equal normal stress and equal speeds on contact surface.

In described method this boundary conditions does not need additional operations, such as "ghost particles" or special integration procedures for (2) to treat a boundary.

Smoothing length $h$ was selected to provide sufficient count of neighbors for each particle for correction matrix to be well defined. Usually neighbor count was from 11 to 16 .

Time step is defined via Courant-Friedrichs-Lewy condition: $\Delta t<\min [h / C]$, where $\mathrm{C}$ is the sound speed. At practice stability condition for heat transfer problem is more weak while droplet radius exceed $1 \mu \mathrm{m}$.

\section{Results}

A $38 \mu \mathrm{km}$ radius droplet of molten stainless steel is impacting with a velocity of $200 \mathrm{~m} / \mathrm{s}$ on stainless steel substrate. Parameters of steel: density $7.87 \mathrm{~g} / \mathrm{cm} 3, \mathrm{E}=200 \mathrm{GPa}, \mathrm{G}=70 \mathrm{GPa}$, $\mathrm{Ypl}=1.2 \mathrm{MPa}$, viscosity of molten steel $5.4 \mathrm{mPa}^{*} \mathrm{~s}$, heat conductivity $28 \mathrm{Wt} / \mathrm{m} / \mathrm{K}$, heat capacity $0.56 \mathrm{~kJ} / \mathrm{kg}$, temperature of melting $1280{ }^{0} \mathrm{C}$, latent heat $84 \mathrm{~kJ} / \mathrm{kg}$, initial temperature of droplet $1380{ }^{\circ} \mathrm{C}$, initial temperature of substrate $0{ }^{0} \mathrm{C}$. 

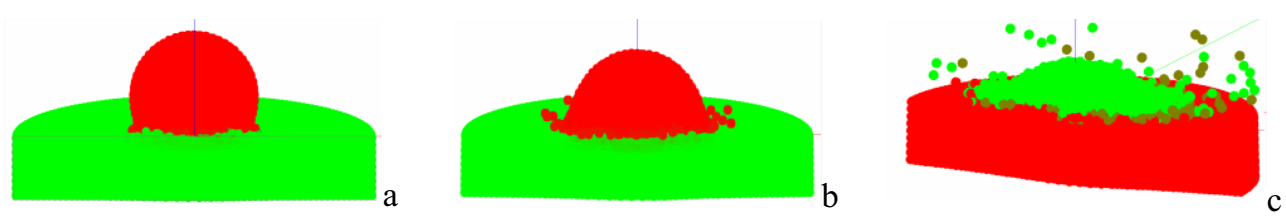

Fig. 1. Impact of molten droplet on substrate. $t=70 \mathrm{~ns}(\mathrm{a}), \mathrm{t}=140 \mathrm{~ns}$ (b), start of solidification at $\mathrm{t}=140$ ns: solidificated points are marked with dark grey, solid- red and melted with lime (c).

As it shown at fig. 1, solidification of melted material starts at contact surface approximately $140 \mathrm{~ns}$ after contact and significant fragmentation of droplet due to high initial speed is observed. Energy conservation during calculation doesn't exceed $0.1 \%$, full change of momentum doesn't exceed $0.01 \%$.

\section{Acknowledgments}

This work was supported by the Russian Science Foundation (RSF) No. 16-19-10264.

\section{References}

1. L.B. Lucy, Astronomical J. 82 (1977)

2. M.B. Liu, G.R. Liu, Applied Numerical Mathematics 56, 1 (2006)

3. M. Biot, J. Math. Mech. 15, 2 (1966) 